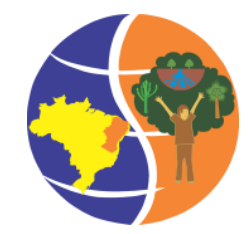

II Simpósio Brasileiro de Recursos Naturais do Semiárido - SBRNS

"Convivência com o Semiárido: Certezas e Incertezas"

Quixadá - Ceará, Brasil

27 a 29 de maio de 2015

doi: 10.18068/IISBRNS2015.biod474

ISSN: 2359-2028

\title{
RENDA E EFICIÊNCIA DE USO DA TERRA NO CULTIVO ORGÂNICO DO GERGELIM COM CULTURAS ALIMENTARES
}

\author{
José Rodrigues Pereira ${ }^{1}$, Érica Samara Araújo Barbosa de Almeida ${ }^{2}$, Paulo de Tarso Firmino ${ }^{3}$, \\ Vinicius Evangelista Alves Oliveira ${ }^{4}$, José Francisco de Sousa Filho ${ }^{5}$ \\ ${ }^{1}$ Dr., Pesquisador, Fitotecnia, Embrapa Algodão, Rua Oswaldo Cruz, 1143 - Centenário, CEP 58.428-095, Campina Grande, \\ PB, 833182 4373, jose.r.pereira@embrapa.br. \\ ${ }^{2}$ Engenheira Agrônoma, Mestranda, Engenharia Agrícola, UFCG, Campina Grande, PB, ericasamara@gmail.com. \\ ${ }^{3}$ M. Sc., Pesquisador, Tecnologia de Alimentos, Embrapa Algodão, Campina Grande, PB, paulo.firmino@embrapa.br. \\ ${ }^{4}$ Graduando em Agronomia, UFPB, Areia, PB, 839609 6785, vinicius_oliver@ hotmail.com. \\ ${ }^{5}$ Téc. Agríc. Extensionista, Fraternidade S. Francisco de Assis - FFA, S. Fco de Assis do Piauí, PI, zefilhosousa@gmail.com.
}

RESUMO: Objetivou-se avaliar a renda bruta auferida por diferentes policultivos/arranjos de plantio e monocultivos envolvendo o gergelim com outras culturas alimentares e a eficiência total de uso da terra desses consórcios quando comparados com monocultivos das culturas utilizadas. $\mathrm{O}$ experimento foi conduzido na Comunidade Veredas, município de São Francisco de Assis do Piauí, Vale do Canindé, PI, em blocos casualizados e em esquema fatorial 3x4, com 3 repetições, envolvendo três sistemas de policultivo $(\mathrm{C} 1$ - Gergelim + Milho; $\mathrm{C} 2$ - Gergelim + Feijão vigna; C3 - Milho + Feijão vigna) e quatro arranjos de plantio das culturas em consórcio (A1 - 1:1; A2 1:2; A3 - 2:1; A4 - 2:2) mais três tratamentos-testemunhas (monocultivos das cultivares BRS Seda de gergelim, BRS Cruzeta de milho e BRS Guaribas de feijão vigna). Foram calculadas a renda bruta e a eficiência total de uso da terra através de equações próprias. Concluiu-se que os policultivos ou consórcios tiveram maior produção que as monoculturas de suas espécies componentes, correspondendo, em média, a 2,5 hectares do cultivo isolado de quaisquer duas das culturas utilizadas na pesquisa, tendo o consórcio Gergelim + Feijão vigna, abrangendo todos os tipos de arranjos de plantio estudados, auferido as maiores rendas brutas e os maiores índices de eficiência total de uso da terra.

PALAVRAS-CHAVE: agroecossistemas, monocultivo, consorciamento

\section{SESAME ORGANIC INCOME AND TOTAL LAND EQUIVALENT RATIO WITH FOOD CROPS}

ABSTRACT: To evaluate the gross income obtained by different intercropping systems/planting patterns and monocroppings involving sesame with other food crops and the total index of land equivalent ratio of these intercropping systems compared to single crops used, an experiment was conducted in the Veredas Community, São Francisco de Assis do Piauí county, Canindé Valley, Piauí State, Brazil, in randomized blocks and in a $3 \times 4$ factorial scheme, with three repetitions, using three intercropping systems $(\mathrm{C} 1$ - Sesame+Corn; $\mathrm{C} 2$ - Sesame+Cowpea bean; $\mathrm{C} 3$ - Corn + Cowpea bean) and four planting patterns of the crops used (A1 - 1:1; A2 - 1:2; A3 - 2:1; A4 2:2) plus three control-treatments (single crops of sesame BRS Seda, corn BRS Cruzeta and cowpea bean BRS Guaribas). The gross income and the total index of land equivalent ratio were calculated by its own equations. It was concluded that the intercropping systems had higher yield that the monocroppings of its component species, corresponding, on average, to 2.5 hectares of single crops of any two crops used in research, and the Sesame+Cowpea bean intercropping system, covering all kinds of studied planting patterns, promoted the highest gross incomes and the highest indexes of total land equivalent ratio.

KEYWORDS: agroecossystems, monocropping, intercropping 


\section{INTRODUÇÃO}

Com a agricultura convencional, o homem simplificou a estrutura do ambiente, trocando a diversidade natural por um pequeno número de plantas cultivadas (monoculturas) e animais domésticos quando se sabe que, a estabilidade de um agroecossistema é tanto maior quanto maior for o número e a diversidade de seus componentes (COUTINHO et al., 2003).

Os sistemas de cultivos tradicionais em pequenas propriedades, com múltiplos cultivos (policultivos), promovem a diversidade de alimentação e recursos, estabilidade de produção, minimização de riscos, redução da incidência de insetos e doenças, uso eficiente da mão-deobra, intensificação da produção com recursos limitados e maximização de recursos sob baixo nível tecnológico (WILLEY, 1979; COUTINHO et al., 2003); adicionalmente, o produtor poderá obter maior rendimento com o melhor aproveitamento dos recursos ambientais (como luz, umidade do solo, nutrientes, etc.) além da vantagem de redução da erosão do solo e, possivelmente, da proteção e suporte que uma cultura poderá oferecer a outra (WILLEY, 1979).

Existe a necessidade de se preservar o conhecimento acumulado e transformado em tecnologias pelos produtores, como forma de preservar o meio ambiente. Para que isto ocorra, defendem a necessidade da extensão rural se ajustar ao processo de introdução de tecnologias e de produtos não-tradicionais, buscando novas alternativas para viabilizar sistemas que melhorem o uso dos recursos e ao mesmo tempo provoquem o incremento da renda dos agricultores familiares (MENEZES, 2002).

O índice de uso eficiente da terra (UET) é definido como a área relativa de terra em condições de plantio isolado, que é requerida para proporcionar os rendimentos alcançados no consórcio. É o indicador mais utilizado para avaliação de sistemas policulturais (WILLEY, 1979).

Esse trabalho teve por objetivos avaliar a renda auferida por diferentes policultivos/arranjos de plantio e monocultivos envolvendo o gergelim com outras culturas alimentares e a eficiência total de uso da terra (UETotal) desses consórcios quando comparados com os respectivos monocultivos.

\section{MATERIAL E MÉTODOS}

O presente estudo foi realizado no município de São Francisco de Assis do Piauí, PI, localizado na microrregião de Alto Médio Canindé. A agricultura praticada na região é baseada na produção sazonal de feijão, milho e arroz (BRASIL, 2006).

O delineamento experimental utilizado foi blocos casualizados em esquema fatorial $3 \times 4$, com 3 repetições, envolvendo 3 sistemas de policultivo (C1 - Gergelim+Milho; C2 - 
Gergelim+Feijão vigna; C3 - Milho+Feijão vigna) e 4 arranjos de plantio das culturas em consórcio (A1 - 1:1; A2 - 1:2; A3 - 2:1; A4 - 2:2) mais três tratamentos-testemunhas, representados pelos monocultivos das culturas envolvidas. Cada parcela mediu 10,0 m x 6,0 m $60 \mathrm{~m} 2$ totalizando $900 \mathrm{~m} 2$ por bloco e $2700 \mathrm{~m} 2$ de área experimental, totalizando $3060 \mathrm{~m} 2$ de área. As culturas, tanto em consórcio como isoladas, foram espaçadas de 1,00 m entre fileiras com densidade $10 \mathrm{plantas} / \mathrm{m}$ de fileira.

O preparo do solo foi feito com arado de aiveca, tracionado por boi. Todos os poli e monocultivos foram plantados no mesmo dia. Foram utilizadas as cultivares BRS Seda de gergelim, BRS Cruzeta de milho e BRS Guaribas de feijão vigna. Foi usada adubação com esterco caprino curtido na base de $20 \mathrm{t}$ ha-1, realizada em área total antes do preparo do solo. A única capina foi feita através de tração animal.

O rendimento de grãos das culturas foi determinado pela pesagem (gramas) das sementes de cada área útil (2 fileiras de $5,0 \mathrm{~m}$ ) da parcela experimental e repetição por respectivo tratamento, convertendo-se para $\mathrm{kg}$ ha-1. A renda bruta auferida para cada tratamento resultou do somatório da multiplicação do(s) rendimento(s) da(s) cultura(s) pelo(s) preço(s) unitário(s) (em Reais - R\$), no mercado local, do quilograma do(s) produto(s) constante(s) no tratamento.

A estimativa da participação de cada cultura agrícola no rendimento combinado em consórcio foi efetuada pelo índice total de uso eficiente da terra (UETotal) calculado pela fórmula (1) sugerida por Willey (1979):

$$
\text { UETotal }=\sum_{i=1}^{m} \frac{Y_{i}}{Y_{i i}}
$$

Onde:

Y $i=$ rendimento da cultura em consórcio, $\mathrm{kg} \mathrm{ha}^{-1}$;

Yii = rendimento da cultura solteira, $\mathrm{kg} \mathrm{ha}^{-1}$.

Os dados médios dos tratamentos estudados nas variáveis renda bruta e eficiência total de uso da terra (UETotal) foram submetidos à análise de variância, a 1 e $5 \%$ de probabilidade (teste F), e ao teste de Tukey, a 5\% de probabilidade, em ambos utilizando-se o software estatístico SISVAR (FERREIRA, 2011).

\section{RESULTADOS E DISCUSSÃO}

Na Tabela 1, conforme a análise de variância, observa-se que houve diferenciação significativa entre os tratamentos estudados em todas as variáveis computadas e analisadas. 
Tabela 1. Quadrados médios da análise de variância da renda bruta $(\mathrm{R} \$)$ e da eficiência total de uso da terra (UETotal) de diferentes policultivos/arranjos de plantio e monocultivos estudados no Vale do Canindé, PI. São Francisco de Assis do Piauí, PI. 2014

\begin{tabular}{llll}
\hline Fonte de variação & GL & Renda Bruta & UETotal \\
\hline Blocos & 2 & $116757,19 \mathrm{~ns}$ & $0,42 \mathrm{~ns}$ \\
Tratamentos & 14 & $8616860,40^{* *}$ & $1,08 * *$ \\
Erro & 28 & 336380,96 & 0,31 \\
\hline Média & - & 4698,11 & 2,07 \\
CV $(\%)$ & - & 12,35 & 27,21 \\
\hline
\end{tabular}

* Significativo $(\mathrm{p}<0,05), * *$ significativo $(\mathrm{p}<0,01)$, ns não significativo $(\mathrm{p}>0,05)$

Aplicado o teste de Tukey, constatou-se que, dentre as sete maiores rendas brutas auferidas, quatro delas ocorreram nos tratamentos envolvendo o consórcio Gergelim + Feijão vigna, notadamente abrangendo todos os tipos de arranjos de plantio estudados. As menores rendas brutas foram apresentadas pelos monocultivos das culturas, a menor de todas sendo a do monocultivo do Milho BRS Cruzeta (Tabela 2). Esse resultado assemelha-se ao obtido por Bhatti et al. (2013) testando o consorciamento do gergelim com uma cultivar de feijão vigna do Paquistão. Jabbar et al. (2010 a,b) complementam que os policultivos promovem sobretudo estabilidade de produção, minimização de riscos e uso eficiente da mão-de-obra e dos recursos.

Observa-se ainda que, comparados aos tratamentos de monocultivo (UETotal $=1,0$ ), dos sete maiores índices de eficiência total de uso da terra (UETotal) obtidos no experimento, os quais variaram de 2,4 a 2,6, três deles apresentaram-se nos tratamentos envolvendo o consórcio Gergelim + Feijão vigna (Tabela 2), correspondendo, na média desses índices, que se necessitariam de 2,5 hectares do cultivo isolado de gergelim ou de milho ou de feijão vigna para se produzir o equivalente a 1,0 (um) hectare do consórcio envolvendo quaisquer duas dessas culturas, corroborando Liebman (2012) que afirma que um consórcio tem maior produção que as monoculturas de suas espécies componentes sempre que UET > 1,0.

Bhatti et al. (2008) afirmam que evidências recentes realmente sugerem os benefícios notáveis de se consorciar leguminosas. Ademais, segundo Wahla et al. (2009), quando leguminosas são cultivadas em associação com não-leguminosas, muitas vezes há vantagem para as não-leguminosas do nitrogênio fixado por aquelas, além de que, conforme Jabbar et al. (2009) complementam, duas culturas diferentes em hábitos adaptação e de crescimento crescem simultaneamente com menos competição.

Bhatti et al. (2013) mencionam que o consórcio é reconhecido como um sistema de produção mais eficiente, não só por proporcionar melhor rendimento por unidade de área, mas 
também por aumentar a eficiência de utilização de recursos e possibilitar uma melhor renda aos agricultores.

Tabela 2. Renda bruta e eficiência total de uso da terra (UETotal) médios de diferentes policultivos/arranjos de plantio e monocultivos estudados no Vale do Canindé, PI. São Francisco de Assis do Piauí, PI. 2014

\begin{tabular}{lll}
\hline $\begin{array}{l}\text { Policultivos/Arranjos } \\
\text { Monocultivos }\end{array}$ & $\begin{array}{l}\text { Renda Bruta } \\
(\mathrm{R} \$ \text { / hectare })\end{array}$ & $\begin{array}{l}\text { UETotal } \\
\text { (Adimensional) }\end{array}$ \\
\hline Gergelim + Milho / 1:1 & $4786 \mathrm{bcde}$ & 2,2 \\
Gergelim + Feijão / 1:1 & $6305 \mathrm{ab}$ & 2,5 \\
Milho + Feijão / 1:1 & $3565 \mathrm{def}$ & 2,1 \\
Gergelim + Milho / 1:2 & $6902 \mathrm{a}$ & 2,6 \\
Gergelim + Feijão / 1:2 & $5850 \mathrm{abc}$ & 2,1 \\
Milho + Feijão / 1:2 & $3624 \mathrm{def}$ & 1,9 \\
Gergelim + Milho / 2:1 & $5252 \mathrm{abcd}$ & 2,4 \\
Gergelim + Feijão / 2:1 & $6647 \mathrm{a}$ & 2,6 \\
Milho + Feijão / 2:1 & $3514 \mathrm{def}$ & 2,0 \\
Gergelim + Milho / 2:2 & $5542 \mathrm{abc}$ & 2,5 \\
Gergelim + Feijão / 2:2 & $6897 \mathrm{a}$ & 2,6 \\
Milho + Feijão / 2:2 & $4437 \mathrm{cde}$ & 2,6 \\
Gergelim & 3359 ef & 1,0 \\
Milho & $1461 \mathrm{~g}$ & 1,0 \\
Feijão & $2331 \mathrm{fg}$ & 1,0 \\
\hline DMS & 1755 & 1,7 \\
\hline
\end{tabular}

Preços no mercado local de Simplício Mendes, PI(julho de 2014): $\mathrm{R} \$ 3,00 \mathrm{~kg}^{-1}$ de gergelim, $\mathrm{R} \$ 0,75 \mathrm{~kg}^{-1} \mathrm{de}$ milho e R $\$ 2,00 \mathrm{~kg}^{-1}$ de feijão vigna.

\section{CONCLUSÕES}

Os policultivos ou consórcios tiveram maior produção que as monoculturas de suas espécies componentes, correspondendo, em média, a 2,5 hectares do cultivo isolado de quaisquer duas das culturas utilizadas na pesquisa;

O consórcio Gergelim + Feijão vigna, abrangendo todos os tipos de arranjos de plantio estudados, auferiram as maiores rendas brutas e os maiores índices de eficiência total de uso da terra. 


\section{REFERÊNCIAS}

BHATTI, I. H.; AHMAD, A. R.; JABBAR, A.; ASLAM, V.; ASLAM, M. Agro-economic performanceof mungbean intercropped in sesame underdifferent planting patterns.Pakistan Journal Agricultural Science, v.45, n.3, p.25-28, 2008.

BHATTI, I. H.; AHMAD, R.; JABBAR, A.; NADEEM, M.; KHAN, M. M.; VAINS, W-D; VAINS, S. N. Agronomic performance of mash bean as an intercrop in sesame under different planting patterns. Emirates Journal of Food and Agriculture, v.25, n.1, p.52-57, 2013.

BRASIL. Companhia de Desenvolvimento dos Vales do São Francisco e do Parnaíba CODEVASF. Plano de ação para o desenvolvimento integrado da bacia do Parnaíba PLANAP. Síntese executiva: Território Vale do Canindé. Brasília: CODEVASF/PLANAF, 2006. 68p.

COUTINHO, H. L. da C.; UZEDA, M. C.; ANDRADE, A. G. de; TAVARES, S. R. de L. Ecologia e biodiversidade do solo no contexto da agroecologia. Informe Agropecuário, v.24, n.220, p.45-54, 2003.

FERREIRA, D. F. Sisvar: a computer statistical analysis system. Ciência e Agrotecnologia, v.35, n.6, p.1039-1042, 2011.

JABBAR, A.; AHMAD, R.; BHATTI, I. H.; DIN; W.; NADEEM, M.; KHAN, M. M. Evaluating the performance of direct seeded rice in different intercropping systems under strip plantation. International Journal Agricultural Biology, v.12, n.04, p.501-508, 2010a.

JABBAR, A.; AHMAD, R.; BHATTI, I. H.; REHMAN, A.; VIRK, Z. A.;VAINS, S. N. Effect ofdifferent rice-based intercropping systems onrice grain yield and residual soil fertility. Pakistan Journal Botany, v.42, n.04, p.2339-2348, 2010 b.

JABBAR, A.; AHMAD, R.; BHATTI, I. H.; VIRK, Z. A.; DIN; W.; KHAN, M. M. Assessment of yield advantages, competitiveness and economic benefits of diversified direct seeded upland rice-based intercropping systems under strip geometry of planting. Pakistan Journal Agricultural Science, v.46, n.02, p.96-101, 2009.

LIEBMAN, M. Sistemas de policultivos. In: ALTIERI, M. A. Agroecologia: bases científicas para uma agricultura sustentável. 3.ed., São Paulo, Rio de Janeiro: Expressão Popular, AS-PTA 2012. p.221-240.

MENEZES, A. J. E. A. de. Análise econômica da "produção invisível" nos estabelecimentos agrícolas familiares no projeto de assentamento agroextrativista Praialta e Piranheira, município de Nova Ipixuna, Pará. 2002, 130 f. Dissertação (Mestrado em Engenharias Familiares e Desenvolvimento Sustentável) - Centro Agropecuário/Núcleo de Estudos Integrados sobre Agricultura Familiar, Universidade Federal do Pará, Belém.

WAHLA, I. H.;AHMAD, R.;ULLAH, E.;AHMAD, A.; JABBAR, A. Competition functions of component crops in Barley-based intercropping systems. International Journal Agricultural Biology, v.11, p.69-72, 2009.

WILLEY, R. W. Intercropping - Its importance and research needs. Part 1. Competition and yield advantages. Field Crop Abstracts, v.32, n.1, p.1-10. 1979. 